\title{
Diacronie
}

Studi di Storia Contemporanea

$\mathrm{N}^{\circ} 31,3$ | 2017

“Le armi della politica, la politica delle armi"

\section{Riflessione: cosa fare dell'anniversario del 1917?}

\section{Valerio Romitelli}

\section{(2) OpenEdition \\ Journals}

\section{Edizione digitale}

URL: http://journals.openedition.org/diacronie/6013

DOI: $10.4000 /$ diacronie. 6013

ISSN: 2038-0925

\section{Editore}

Association culturelle Diacronie

Notizia bibliografica digitale

Valerio Romitelli, «Riflessione: cosa fare dell'anniversario del 1917? », Diacronie [Online], №31,

3 | 2017, Messo online il 29 octobre 2017, consultato il 30 avril 2019. URL : http://

journals.openedition.org/diacronie/6013; DOI : 10.4000/diacronie.6013 


\section{Diacronie}

Studi di Storia Contemporanea

$31,3 / 2017$

"Le armi della politica, la politica delle armi". Ideologie di lotta ed esperienze di guerra

\section{Riflessione: Cosa fare dell'anniversario del 1917?}

\section{Valerio ROMITELLI}

Per citare questo articolo:

ROMITELLI, Valerio, «Riflessione: Cosa fare dell'anniversario del 1917?», Diacronie. Studi di Storia Contemporanea : "Le armi della politica, la politica delle armi". Ideologie di lotta ed esperienze di guerra, 31, 3/2017, 29/10/2017,

URL: < http://www.studistorici.com/2017/10/29/romitelli_numero_31/ >

Diacronie Studi di Storia Contemporanea $\rightarrow$ http://www.diacronie.it

Rivista storica online. Uscita trimestrale.

redazione.diacronie@hotmail.it

Comitato di direzione: Naor Ben-Yehoyada - João Fábio Bertonha - Christopher Denis-Delacour - Maximiliano Fuentes Codera Anders Granås Kjøstvedt - John Paul Newman - Deborah Paci - Niccolò Pianciola - Spyridon Ploumidis - Wilko Graf Von Hardenberg

Comitato di redazione: Jacopo Bassi - Luca Bufarale - Gianluca Canè - Fausto Pietrancosta - Alessandro Salvador - Matteo Tomasoni Diritti: gli articoli di Diacronie. Studi di Storia Contemporanea sono pubblicati sotto licenza Creative Commons 3.0. Possono essere riprodotti e modificati a patto di indicare eventuali modifiche dei contenuti, di riconoscere la paternità dell'opera e di condividerla allo stesso modo. La citazione di estratti è comunque sempre autorizzata, nei limiti previsti dalla legge. 


\title{
Riflessione/ Che fare dell'anniversario del 1917?
}

\author{
Valerio ROMITELLI
}

A cent'anni dalla Rivoluzione d'Ottobre, ci si trova di fronte a interrogativi e riflessioni sull'eredità di un evento che ha cambiato radicalmente il volto del XX secolo. Il crollo del socialismo reale si è portato appresso uno stigma che ha reso difficile un'analisi critica e possibilmente priva di pregiudizio sulla portata effettiva del 1917 e sul grande periodo di cambiamenti sociali e movimenti emancipatori che essa ha innescato.

Questo contributo, senza aspirare ad una esaustiva rassegna storiografica, vuole mettere in luce i principali nodi della riflessione sul 1917 e le sue conseguenze di lungo periodo prendendone in considerazione analisti e critici e cercando di trarre alcune conclusioni sulla pesante eredità della più importante rivoluzione della contemporaneità.

Ci sono centenari dall'immensa portata simbolica. Caso clamoroso da tale punto di vista è quello della Rivoluzione Francese del 1789: che nell'Ottocento costitù̀ un'importante occasione per il formarsi della socialdemocrazia in Europa e che invece nel Novecento registrò il disfarsi dell'Unione sovietica col conseguente crollo di credibilità di tutto il comunismo. Ben diversamente il centenario in corso della Rivoluzione dell'ottobre 1917 in Russia, fino a imprevedibili prove contrarie, non pare sancire alcunché di simbolicamente rilevante.

Al suo cadere c'è comunque da rilevare quanto l'immagine del suo evento originario si sia trasformata anche solo rispetto ad una trentina d'anni fa. In effetti, da allora ad oggi la teoria critica del totalitarismo che aveva preso slancio in piena guerra fredda ha finito per trionfare coniugandosi perfettamente con le dottrine della globalizzazione neoliberista. Così l'assimilazione tra hitlerismo e stalinismo, lungi dal far scandalo, è attualmente un dato quasi obbligatorio dell'opinione corrente, di qualsiasi orientamento politico. Di qui l'imporsi dell'obbligo di giudicare con riserve e sospetti, se non di condannare senza appello ogni politica passata o presente avente a riferimento comunismo e marxismo. 
Caratteristica peculiare di questo centenario è dunque quella di cadere in un contesto culturale e intellettuale per lo più mondialmente ostile ${ }^{1}$. Certo si può giustamente insistere nel denunciare l'anticomunismo particolarmente aggressivo della cultura neoliberale dominante. Ma in tal modo si rischia di mancare una questione ben più rilevante. Quella, per dirla col bel titolo di un libro di qualche anno fa, del «silenzio dei comunisti» ${ }^{2}$. Ossia della mancanza a tutt'oggi di un bilancio e di un'autocritica, da parte di chi ha creduto nell'idea comunista, all'altezza del tracollo della sua credibilità a partire dal 1989. Una mancanza aggravata dal fatto che le difese del comunismo e anche di ciò che più genericamente si insiste a chiamare "sinistra" avvengono per lo più su un piano etico, in nome di molto spesso poco chiari "valori" che si manterrebbero sempre identici a loro stessi perché supposti di levatura trascendente, dunque al di sopra di ogni accadimento storico. È anche per questo che, come accade in ogni sconfitta non dichiarata e non ripensata come tale, anche quella sancita dal 1989 si è trasformata in una disfatta progressivamente estesasi fino alle sue ultime conseguenze. Basti pensare alla raffinatezza delle diatribe teoriche ed epistemologiche che hanno accompagnato tutta la storia di socialisti e comunisti fino agli Settanta confrontata alla modestia intellettuale che è prevalsa da allora in poi tra chi ha provato $\mathrm{a}$ continuarne la tradizione. Fino al punto che personalità di spicco della sinistra sono notoriamente divenuti i più efficaci paladini del neoliberalismo. Né è molto proficuo ostinarsi a prendersela con il generale abbassamento culturale indotto dai media o da internet. C'è piuttosto da chiedersi come mai in nome del comunismo da una trentina di anni in qua non si sia trovato nulla capace di contrastare questo abbassamento. La conclusione da trarre a questo proposito mi pare possa essere una sola: che il comunismo ha esaurito la sua forza ideale e di sperimentazione politica; o, detto più brutalmente, il comunismo è davvero finito. Col che non è escluso che tale parola possa risorgere, ma così come non è neanche da escludere che si sia giunti ad un punto simile a quello che nel corso del 1917 fece decidere i bolscevichi di abbandonare il nome di socialdemocratici perché appunto aveva secondo loro finito per perdere ogni forza ideale e di sperimentazione

\footnotetext{
${ }^{1}$ Così non pare leggendo uno dei pochi libri che hanno il grande merito di aver colto l'occasione centenaria per ritrattare dell'ottobre 1917, sia pur descritto nel contesto molto più ampio dei maggiori accadimenti capitati in quell'anno a livello planetario. In D'ORSI, Angelo, 1917 L'anno della Rivoluzione, Roma-Bari, Laterza, 2017, la classica versione di questa rivoluzione come conseguenza storica necessaria delle immani sofferenze prodotte dalla prima guerra mondiale viene infatti esposta in modo così scorrevole da apparire di un'evidenza quasi incontestabile. Un altro recente testo che non sembra risentire troppo del clima culturale ostile è il decisamente nostalgico FERRANDO, Marco, Cento anni. Storia e attualità della rivoluzione comunista, Roma, Redstarpress, 2016. Per il resto, a mia conoscenza, la bibliografia disponibile in italiano su questo argomento è comunque datata. Tra i principali si veda BUDGEN, Sebastian, KOUVELAKIS, Stathis, ŽIŽEK, Slavoj (a cura di), Lenin 2.0. La verità è di parte, Milano, Transeuropaedizioni, 2008; FLORES, Marcello, 1917. La Rivoluzione, Torino, Einaudi, 2007; GRAZIOSI, Andrea, L'Urss di Lenin e Stalin. Storia dell'Unione sovietica 1914-1945, Bologna, Il Mulino, 2007; PIPES, Richard, La rivoluzione russa. Dall'agonia dell'Ancien Règime al terrore rosso, Milano, Mondadori, 1995; ANWEILER, Oskar, Storia dei soviet. I consigli di fabbrica in Urss. 1905-1921, Roma-Bari, Laterza, 1972; ROSENBERG, Arthur, Storia del bolscevismo, Firenze, Sansoni, 1969; CARR, Edward H., La rivoluzione bolscevica. 1917-23, Torino, Einaudi, 1964.

${ }^{2}$ FOA, Vittorio, MAFAI, Miriam, REICHLIN, Alfredo, Il silenzio dei comunisti, Torino, Einaudi, 2002.
} 
politica. La discussione in merito trovo sia comunque da lasciare aperta, ma quel che sarebbe il caso di chiudere è la supposizione etico-filosofica secondo la quale il comunismo o la "sinistra" restino valori trascendenti, eterni, incorruttibili al punto di potere essere custoditi nel proprio animo, qualsiasi cosa accada nel mondo ${ }^{3}$. Per un pensiero politico sperimentale, quale quello che mi sforzo da anni di elaborare, vale tutta un'altra ottica, del tutto immanente, che prende come orizzonte il divenire colto nel suo tempo presente, all'incrocio tra un bilancio del passato e una possibile agenda per l'avvenire. Di qui, l'ipotesi, sia pur tutta da dimostrare, che non sia completamente vano rivendicare un approccio dichiaratamente post-comunista.

Il comunismo o la sinistra supposte come idee trascendenti implicano spesso un'altra supposizione parimenti contestabile: quella secondo la quale pur restando sempre validi i principi democratici ed egualitari occorrerebbe constatarne il loro fallimento nelle esperienze del "socialismo reale". Si tratta in altre parole della convinzione secondo la quale il comunismo come ideale resta sempre plausibile anche se in realtà ha fallito. Anche qui ad avviso di chi scrive si tratta invece di rovesciare il discorso. Tutto al contrario sono convinto infatti che si debba constatare che se il comunismo è finito, non per questo ha fallito. Anzi come sperimentazione politica credo occorra ammettere che in suo nome è stato raggiunto il massimo risultato mai raggiunto da precedenti sperimentazioni politiche. Il risultato consistente nell'avere favorito l'epoca storica più propizia alla riduzione delle disparità sociali, sarebbe a dire "i trent'anni gloriosi", grosso modo tra il 1945 e il 1975. Si può in effetti discutere quanto si vuole sui limiti e i costi comportati dalla tendenza dominante in questa epoca, ma resta comunque che essa ha favorito fenomeni del tutto contrari a quelli che oggi affliggono il mondo, in termini soprattutto di diseguaglianze tra ricchi e poveri, nonché di falcidie del ceto medio. Salari alti, assistenza e istruzione universali, ma anche emergere del "terzo mondo" come protagonista politico e così via: questa, dunque, la tendenza globalmente operante in assoluta contemporaneità all'espansione in almeno metà del pianeta di regimi fatti ad immagine dell'Urss. Come credere che tale contemporaneità sia stata una pura coincidenza?

Piuttosto, si può obiettare che a riconoscere i successi dell'Urss e del diffondersi planetario del suo modello si rischia comunque di sminuire gli orrori connessi. Insomma come pretendere di sconfessare tutto dei tanti "libri neri" del comunismo? Non è questo il problema. Si può perfettamente ammettere, ad esempio con Solženicyn, che l'immensa Santa Madre Russia si sia immolata tramite le pene inflitte da Stalin per alleviare i peccati di ingiustizia dell'intera

\footnotetext{
${ }^{3}$ Un'opinione questa che può trovare sostegno anche da testi del tutto distanti tra loro come ad esempio: BOBBIO, Norberto, Destra e sinistra. Ragioni e significati di una distinzione politica, Roma, Donzelli, 1995 e BADIOU, Alain [et al.], DOUZINAS, Costas, ŽIŽEK, Slavoj (a cura di), L'idea di comunismo, Roma, DeriveApprodi, 2011.
} 
umanità ${ }^{4}$ : ciò non toglie che, da un punto di vista meno misticheggiante, così si è avuto un effetto di contenimento degli "spiriti animali" del capitalismo, che oggi non si riesce più a creare, mentre il mondo è sempre più dilaniato dall'accrescersi delle differenze sociali. Del resto anche se su un piatto del giudizio storico poniamo le enormi cifre delle vittime della "costruzione del socialismo in un paese solo", sull'altro piatto non si possono non mettere i 23 milioni di morti tra partigiani, soldati e gente comune mobilitati dalla "grande guerra patriottica" e che ha finito per bloccare irreversibilmente il nazismo a Stalingrado. Insomma anche il discutibile calcolo delle vittime non arriva a cancellare i successi ottenuti dall'Urss, che possono essere negati solo per scelta pregiudiziale.

Ma se ammettiamo che le sue maggiori riuscite il comunismo le ha conosciute grazie al regime uscito dell'ottobre 1917, allora si comprende come sia il caso di provare a reinterrogare questo evento per capire da dove siano venute le virtù di questo regime e di tutte le sue successive imitazioni, nonostante la loro conclusione una trentina d'anni fa. Quanto poi a tale conclusione, una volta constatata, val la pena di chiedersi se essa sia dipesa da un vizio di origine sempre risalente all'ottobre 1917 oppure sia stato generato da tradimenti o revisioni successive, quali quelli che Trockij imputava a Stalin o ai maoisti ai comunisti di osservanza sovietica e così via. La risposta a tale questione dipende da come si valuta tale fine. Se la si considera concernente l'insieme di tutte le esperienze condotte in nome di marxismo e comunismo, come qui propongo, allora è chiaro che da reinterrogare è proprio l'evento a partire dal quale "tutto ebbe inizio", ossia l'ottobre 1917.

Virtù e vizi di questa origine: ecco dunque un tema niente affatto obsoleto e quanto mai opportuno per celebrare questo centenario. Così ho tentato in effetti di fare con un opuscolo di prossima uscita, L'enigma dell'ottobre 1917. Perché ripensare oggi la "rivoluzione russa", senza rivendicare alcuna qualifica di esperto di storia russa, ma presentando una sintesi degli ormai innumerevoli anni passati in dibattiti, riflessioni e tormenti intorno a questo evento cruciale per ogni militante comunista ${ }^{5}$.

Senza qui anticiparne le argomentazioni principali mi limito a segnalarne alcuni nodi problematici:

- anzitutto la controversa vicenda dei rapporti tra soviet, bolscevichi e gli altri partiti tra il 1905 e il 1917, fino alla svolta dell'ottobre;

- la svolta che questa data ha significato nella stessa storia del bolscevismo modificando radicalmente questo partito;

\footnotetext{
${ }^{4}$ SOLŽENICYN, Aleksandr, Arcipelago Gulag, 1918-'56. Saggio di inchiesta narrativa, Milano, Mondadori, 1978.

${ }^{5}$ ROMITELLI, Valerio, L'enigma dell'ottobre 1917, Napoli, Edizioni Cronopio, 2017.
} 
- lo scioglimento dell'Assemblea Costituente, la pace di Brest-Litovsk, la Prima internazionale, il "Comunismo di guerra", la NEP, come momenti salienti della politica del governo bolscevico;

- l'eredità dell'ottobre 1917 e le sue metamorfosi nel corso della "costruzione del socialismo in un paese solo";

- la contraddittorietà del pensiero di Lenin spesso debordante nel corso degli eventi oltre i suoi stessi presupposti dottrinari;

- la questione dello statuto epistemologico oltre che politico di tale pensiero.

Un nodo problematico, quest'ultimo, che merita qui qualche annotazione supplementare. In effetti nel porlo mi sono ispirato a quella corrente dell'epistemologia francese (Bachelard, Kore, Foucault, tra gli altri) che ha costituito un riferimento maggiore di quella che può essere considerata l'ultima filosofia dichiaratamente marxista, quella di Louis Althusser ${ }^{6}$. Una scelta non casuale questa, ma dovuta ad una precisa ragione. Essa sta nella rielaborazione allargata da parte di Althusser tra gli anni Sessanta e Settanta di quello che era stato, più di mezzo secolo prima, uno dei principali oggetti polemici dello stesso Lenin, sarebbe a dire l'“economicismo". Oggetto polemico quanto mai cruciale nella biografia intellettuale di quest'ultimo il quale ad esso dedicò quello che non solo è stato consacrato come uno dei testi più classici dei cosiddetti classici del marxismo, ma che può anche essere ritenuto il testo fondatore del bolscevismo. Si tratta di quel Che Fare? del 1902 dove Lenin denunciava l'“economicismo", il "tradunionismo", come uno dei problemi che più ostacolavano l'unione e la crescita della socialdemocrazia russa la quale di lì a poco doveva dividersi tra menscevichi e bolscevichi ${ }^{7}$.

Detto in poche e scarne parole, l'aspetto dell'"economicismo" che creava più problemi, da questo punto di vista, era il far credere ai proletari la possibilità di miglioramenti nelle condizioni di vita, i quali invece dovevano essere mostrati impossibili fino a che il regime zarista non fosse stato abbattuto. Morale della favola, per Lenin, era che il primo compito dei socialdemocratici russi di quel tempo stesse nell'elevare la coscienza dei proletari fino a farla divenire sempre e comunque "antagonista" al regime zarista. E a tal scopo un nodo cruciale di tutta l'argomentazione del Che fare? era che la coscienza rivoluzionaria non poteva nascere spontaneamente all'interno dei movimenti popolari, ma poteva venire solo "dall'esterno" di essi, grazie ad elaborazioni teoriche di intellettuali e militanti professionisti.

È da qui che Althusser prenderà spunto per proporre categorie che faranno discutere come "pratica teorica", ma da notare è soprattutto che la sua rielaborazione della polemica anti

\footnotetext{
${ }^{6}$ Principali testi di riferimento qui sono: ALTHUSSER, Louis, Lenin e la filosofia, Milano, JacaBook, 1969; ID., Umanesimo e stalinismo. I fondamenti teorici della deviazione staliniana, Bari, De Donato, 1973; ID., Per Marx, Roma, Editori Riuniti, 1974; MORFINO, Vittorio, PINZOLO, Luca (a cura di), Sul materialismo aleatorio, Milano, Unicopli, 2000.

${ }^{7}$ LENIN, Vladimir Ilic, Che fare? I problemi scottanti del nostro movimento, Roma, Ed. Riuniti, 1974.
} 
economista le conferirà una portata epistemologica senza precedenti. Misurando a dovere la sua estensione si comprende che tale polemica non coinvolge solo una categoria come "spontaneità", ma anche quella di "interesse economico della classe operaia". Di più, mina alla radice l'idea stessa che la storia abbia soggetto e fine/i. In effetti, Althusser porterà l'anti economismo di Lenin fino alla sua estreme conseguenze, fino a rimettere radicalmente in discussione sia l'idea di Storia con la esse maiuscola, sia l'idea di Umanità, come suo supposto soggetto - di cui il proletariato avrebbe dovuto essere agente di realizzazione ultima, essenziale, comunista. L'antieconomicismo diventa così anche antistoricismo e antiumanismo. Così pare andare all'aria tutto o quasi dell'impalcatura concettuale del marxismo più canonico, di quel marxismo per il quale, una volta che la classe operaia cominciasse a contare nella storia e a divenire cosciente del suo interesse economico, essa prima o poi avrebbe portato l'umanità intera a trasformazioni sociali sempre più egualitarie.

Una simile rimessa in discussione di Marx tramite un ripensamento di Lenin da parte di Althusser può far venire in mente un precedente di tutto rispetto. Si tratta di quel noto articolo del giovane Gramsci nel quale egli pochi mesi dopo la rivoluzione d'ottobre per omaggiarla la chiamava, nientemeno, La rivoluzione contro il Capitale, arrivando addirittura a attribuire ai bolscevichi e rivendicare per sé il rinnegamento di Marx ${ }^{8}$ ! Il problema qui è però che, a differenza di Althusser, il discorso sul rapporto tra Lenin e Marx resta più vago. Dopo le prime frasi decisamente blasfeme, tanto più in quanto scritte sull'«Avanti!» organo del partito più marxista dell'Italia di allora, l'articolo di Gramsci sembra quasi far marcia indietro. Vi si può leggere infatti che con l'ottobre '17 ad essere sconfessata e ripudiata è stata solo un certo tipo di lettura dogmatica, positivista, evoluzionista di Marx, mentre tramite tale rivoluzione sarebbe stato pienamente realizzato quanto nella stessa opera di Marx c'era di «spirito vivificatore... continuazione del pensiero idealistico italiano e tedesco». Tesi, antitesi e sintesi, dunque: il marxismo socialdemocratico contraddetto dalla Rivoluzione d'ottobre ma da essa rilevato in una sintesi purificante. Insomma, una discontinuità funzionale ad una continuità che progredisce verso il meglio.

Analoga sarà la posizione dello stesso Lenin e della Internazionale comunista quando, ad esempio, sosterranno che l'epoca aperta dall'ottobre 1917 era quella delle rivoluzioni proletarie vittoriose, dopo l'epoca, quella di Marx, delle rivoluzioni proletarie tentate. Anche qui si coglie bene la volontà di dimostrare che la discontinuità introdotta dall'ottobre 1917 non faceva che confermare e rilanciare più avanti la continuità con Marx. A far ragionare così Lenin e Gramsci, e con essi la moltitudine dei loro compagni, è evidentemente l'intenzione di dimostrare la massima coerenza nel loro pensare e nel loro agire. Ma è evidente anche che a tal scopo essi trovavano le

\footnotetext{
${ }^{8}$ URL: <https://www.marxists.org/italiano/gramsci/17/rivoluzionecontrocapitale.htm > [consultato il 21 settembre 2017].
} 
istruzioni più adeguate facendo riferimento alla logica hegeliana, a quella logica hegeliana notoriamente rivendicata dallo stesso Marx.

Senza pretendere qui di addentrarmi nelle classiche ed interminabili questioni del rapporto tra marxismo e hegelismo mi limito a notare un vantaggio euristico che offre la distanza sempre cercata da Althusser da qualsiasi retaggio di hegelismo. Tale distanza infatti permette di interrogare il rapporto tra Marx e Lenin non solo e non tanto come problema di continuità o meno, di tradimento, rinnegamento o fedeltà, ma anzitutto come problema di differenziazione più o meno progressiva. Qui si fa valere più che mai l'influenza dell'epistemologia francese che Althusser stesso rivendicava come riferimento imprescindibile. In effetti, da questo punto di vista, come dimostrano testi come Dal mondo chiuso all'universo infinito, Studi galileiani di Kore o Le parole e le cose di Foucault ${ }^{9}$, solo per fare qualche esempio, nella storia delle scienze sperimentali il cambiamento o lo sconfinamento di problematica, intenzionalmente perseguiti o indotti da equivoci o fraintendimenti, giocano un ruolo decisivo: a volte persino superiore dei loro più appropriati e coerenti approfondimenti. Ripensare il rapporto tra Marx e Lenin da questo sbieco del cambiamento di problematica è un suggerimento che si può trarre appunto da tutta l'opera di Althusser, anche se egli stesso non si è mai dichiarato esplicitamente in questo senso.

Suo grande gesto strategico è stato anzitutto quello di promuovere il pensiero di Marx al rango del pensiero scientifico moderno; di sfrondare i suoi testi di ogni determinismo economicistico, di ogni escatologia storicistica e di ogni ideologia umanistica per potervi individuare la scoperta di un "continente" inesplorato della conoscenza: quello del materialismo storico, ripensato come scienza della storia delle formazioni sociali, dei suoi confini, dei suoi orizzonti, delle sue leggi. Lenin, invece, Althusser (oltre a riconoscerlo come continuatore della scienza del materialismo storico) lo ha interpellato soprattutto in rapporto alla filosofia, o meglio per mostrare come il leader bolscevico abbia saputo esercitare "la lotta di classe a livello teorico". Due modi diversi dunque, quelli adottati da Althusser ora per affrontare Marx ora per affrontare Lenin. Il primo concernente un campo problematico scientifico, il secondo il campo problematico della lotta di classe a livello più cosciente. A livello simbolico se ne potrebbe dedurre che invece del classico marxismo-leninismo, col trattino di unione, sarebbe da ripensare ad un "marxismo/leninismo", con una barra di disgiunzione che comunque mantiene la prossimità tra i due termini.

Poiché la filosofia per Althusser non ha storia né oggetti di conoscenza, non essendo per lui in fondo altro che un'eterna lotta tra materialismo e idealismo, ci si potrebbe convincere che il pensiero di Lenin, per come lo intende lui, sia riducibile ad una sorta di "antifilosofica", per usare

\footnotetext{
${ }^{9}$ Rispettivamente: KOYRÉ, Alexandre, Dal mondo chiuso all'universo infinito, Milano, Feltrinelli, 1981; ID., Studi galileiani, Torino, Einaudi, 1976; FOUCAULT, Michel, Le parole e le cose. Un'archeologia delle scienze umane, Milano, Rizzoli, 1967.
} 
un termine ampiamente elaborato da Alain Badiou ${ }^{10}$. Cioè un tipo di pensiero che pur rifiutando di costruire un proprio sistema filosofico si impegna, a volte anche proficuamente, nel mettere in crisi quelli di altri. Ma certo non ci si può contentare di simili definizioni a proposito del massimo dirigente della Rivoluzione d'ottobre. Quanto allo stesso Althusser non è senza importanza notare che egli non abbia mai commentato questo evento, nonostante la sua clamorosa importanza. Si potrebbe supporre che ciò sia dovuto alle difficoltà per lui di situare nel suo stesso sistema filosofico qualcosa come un pensiero politico sperimentale. Qualcosa cioè che non è classificabile né come scienza, né come filosofia: qualcosa che non ha oggetto come la filosofia, ma che ha e scandisce una sua storia come avviene per le scienze sperimentali.

Comunque è da qui che si può partire per cercare di inquadrare la novità epistemologica del pensiero di Lenin, almeno a partire dal Che fare? Si tratterebbe dunque di un modo di pensare singolare eppur universale, senza oggetto di conoscenza, ma in grado di sperimentare qualcosa che scandisce la storia, così come le immense conseguenze dell'ottobre 1917 hanno dimostrato. Senza neanche tentare qui di riassumere la questione quanto mai complessa basti notare due novità rispetto ai precedenti più moderni del pensiero politico di cui Machiavelli può sicuramente essere considerato precursore. Queste due novità sono costituite dai due corpi che per Lenin sono condizione necessaria per un'azione politica rivoluzionaria. Da un lato, un preciso corpo dottrinario, i testi Marx ed Engels, da riesaminare e rettificare in base alle esigenze di ogni «analisi concreta di una situazione concreta»; dall'altro, un altrettanto preciso corpo, questo del tutto reale, costituito da un'organizzazione di militanti "professionisti" capaci di utilizzare il marxismo come "guida per l'azione". Entrambe queste condizioni, si noti, evidentemente ignote agli stessi Marx ed Engels, i quali anche sul piano organizzativo, volendo evitare in ogni modo di rientrare tra i ranghi delle diverse sette tirannicide del loro tempo, si erano dovuti limitare a sostenere Leghe e Associazioni eminentemente intellettuali (quale sempre restò in fondo la stessa Associazione Internazionale dei Lavoratori).

Sarebbero dunque anzitutto queste due precise condizioni, intellettuale, l'una, materiale, l'altra, che avrebbero fatto del pensiero di Lenin e dei bolscevichi un pensiero politico sperimentale: sarebbe a dire capace di mettere alla prova, verificare o falsificare le proprie ipotesi alla luce di un'esperienza rigorosamente specificata in termini intellettuali e materiali. E sarebbero proprio la sottovalutazione della prima condizione e il rigetto della seconda ciò che avrebbe caratterizzato tutti i socialdemocratici, come in Russia i socialisti rivoluzionari e menscevichi, che si opposero ai bolscevichi vedendo non di rado in Lenin qualcosa come un "pazzo autoritario". Interessante ricordare che lo stesso Gorkij, poi sostenitore del regime di

\footnotetext{
10 BADIOU, Alain, San Paolo. La nascita del'universalismo, Napoli, Cronopio, 1999; ID., Lacan. Il Seminario. L'antifilosofia 1994-1995, Napoli-Salerno, Orthotes Editrice, 2016; ID., Nietzsche. L'antiphilosophie (séminaire 199192), Paris, Fayard, 2015.
} 
Stalin, nel corso del 1917 imputava a Lenin e ai bolscevichi la colpa prima di voler trascinare tutta la Russia in un esperimento da loro stessi predisposto ${ }^{11}$.

Può apparire strano considerare il pensiero di Lenin senza oggetto. Tale può sembrare che per lui fosse il potere o se si preferisce i rapporti di forza nella lotta di classe. Ma ragionando così ci si preclude la possibilità di capire uno dei caratteri più innovatori di questo modo di sperimentare una politica comunista. Sarebbe a dire il paradosso di quella che si potrebbe anche chiamare con termine di Nietzsche, la volontà di potenza di Lenin e bolscevichi; una volontà di potenza, sì, volta però al depotenziamento del potere e della forza laddove si stavano più concentrando. Così come risulta evidente, ad esempio, dal fatto che uno degli obiettivi primi della presa del Palazzo d'Inverno era proprio sottrarre la Russia dal novero delle potenze belligeranti. Ma analogo significato lo si può riscontrare nella maggior parte delle più famose formule e parole d'ordine che precedono e seguono questo evento. Che vuol dire infatti che guerra e imperialismo sono la stessa cosa, come fa insistentemente Lenin dal 1914 rivolgendosi a tutti socialdemocratici europei, se non provare a demotivarli dalla tentazione di rifugiarsi sotto l'ala protettiva di un fronte o di un altro? Minare la fiducia in ciò che più sembra più potente, dunque rassicurante, è suo motivo ricorrente. Si pensi al tema del "dualismo di potere" che egli lancia con le famose Tesi di aprile in quella Russia post-zarista in cui tutti o quasi non speravano in altro che sottomettersi all'autorità legittima di un nuovo Stato riunente governo e soviet. Si pensi alla parola d'ordine "tutto il potere ai soviet!" lanciata in un momento in cui i primi a non volerne sapere erano gli stessi soviet, diretti com'erano da una maggioranza socialista rivoluzionaria e menscevica fiduciosa solo in un'Assemblea Costituente che legittimasse la spartizione del loro potere con quello di altre istituzioni. E da ultimo si pensi anche al fatto che la dittatura del proletariato, negli auspici di Lenin, lungi dal dover essere uno Stato "organico" - come ne parlerà lo stesso Gramsci -, avrebbe dovuto consistere in uno Stato "a metà", in quanto privo proprio di quel potere militare e di polizia da affidare (à la suisse) al popolo in armi ${ }^{12}$. Nella lingua del leader bolscevico ogni oggetto politico, ogni concentrato di potere, appare così fendersi, spaccarsi per far intravedere un possibile, un insieme di possibilità altrimenti latenti, che si offrono all'azzardo dell'intervento militante.

E poi? Ne L'enigma dell'ottobre '17 avanzo anche un sommario bilancio del "dopo", fino a Stalin. Qui seguendo il filo delle riflessioni ora condotte mi limito a proporne alcune ulteriori a proposito del corpo organizzativo dei bolscevichi.

\footnotetext{
${ }^{11}$ Vedi STRADA, Vittorio, La rivoluzione svelata. Una lettura nuova dell'Ottobre 1917, Roma, Liberal edizioni, 2007.

12 I riferimenti si trovano ovviamente nelle Opere complete di Lenin, (LENIN, Vladimir Ilic, Opere complete, Roma, Ed. Riuniti, 1955-1970), soprattutto nei voll.: XXIII (agosto 1916-marzo 1917), 1965; XXIV (aprilegiugno 1917), 1966; XXV (giugno-settembre 1917), 1967; XXVI (settembre 1917-febbraio 1918), 1966.
} 
Punto chiaro è che già nell'estate del 1917 l'afflusso in esso di truppe in diserzione lo alterano profondamente. Con la costituzione del Comitato Rivoluzionario Militare pochi giorni prima dell'insurrezione il regime soggettivo che si viene configurando è quello di una milizia armata con modi di pensare e di agire ben diversi da quelli vigenti negli anni precedenti, quando Lenin e Trockij erano in esilio e i bolscevichi esistevano quasi solo come frazione minoritaria all'interno dei soviet. Una volta che tra di essi le parole passano alle armi è inevitabile che non restino le stesse come pure i pensieri che le accompagnano e i modi di decidere che ne conseguono. Le vicissitudini del governo che segue l'ottobre 1917 fanno capire che il corpo organizzativo bolscevico, un tempo fatto di soli militanti, si stava contaminando con l'afflusso di figure con un preponderante profilo da impiegato, da funzionario o da soldato regolare. La dice lunga in proposito la vicenda della guerra civile nel corso della quale Trockij coopta nei comandi dell'Armata Rossa alti ufficiali già zaristi. Da partito sempre contiguo ad altri simili come i menscevichi e i socialisti rivoluzionari, i bolscevichi con l'estate 1917 e soprattutto dopo si impongono sempre più come partito-Stato.

Osservando l'insieme di questo processo dal punto di vista della pace di Brest-Litovsk e delle sue condizioni, che Lenin faticò anche tra i suoi compagni a far accettare, lo scandalo sollevato anche allora a proposito del contemporaneo soffocamento del pluralismo dei partiti e della stampa, risulta in gran parte ozioso. Valutando realisticamente la situazione del momento risulta infatti evidente che nessuna maggioranza "democraticamente" espressa avrebbe infatti avallato mai questo gesto che invece garantì a buona ragione il massimo di popolarità a livello mondiale dell'ottobre 1917: solo così i bolscevichi si dimostrarono al mondo come l'unica forza politica davvero capace di mantenere le sue promesse tra le quali anzitutto l'interruzione unilaterale della carneficina della prima guerra mondiale. Ma ancora prima, di fronte al tentato colpo di Stato reazionario di Kornilov, difficile pensare che esso non sarebbe riuscito se Kerenskij avesse fatto appello ai soviet e ai suoi partiti maggioritari, senza ricorrere, come effettivamente, fece all'aiuto principale delle truppe seguaci del partito di Lenin.

In sintesi, contro ogni retorica storicista e trionfalista non si può non riconoscere il carattere minoritario dell'iniziativa bolscevica che ha portato all'ottobre 1917. Invece di pensare che si sia trattato di un evento nel quale si è espresso l'afflato verso il comunismo del gigantesco popolo russo, si deve ammettere che si è trattato piuttosto di un esperimento "da specialisti" che ha introdotto politicamente e militarmente nella storia russa una prospettiva inattesa $\mathrm{e}$ controcorrente.

Che poi a realizzarla sia stata una figura diversa da quella che l'aveva preparata, un PartitoStato oramai bolscevico solo più di nome che di fatto, non deve far pensare ad una semplice degenerazione "autoritaria". Più proficuo dal punto di vista della storia del pensiero politico è valutare quanto questa figura sarà presa a modello dal mondo intero, condizionando non solo i 
destini di Italia e Germania, dirette come saranno da partiti unici chiaramente ispirati al modello bolscevico, ma anche degli Stati Uniti, se è vero che è al partito democratico di Roosevelt (al governo, come Hitler, ininterrottamente dal 1933 al 1945) che si deve non solo la grande svolta del New Deal, ma anche la partecipazione americana alla seconda guerra mondiale, con tutte le enormi conseguenze del caso.

Un protagonismo straordinario in tutto il XX secolo dunque quello da riconoscere all'ottobre 1917: sia a quanto lo ha reso possibile, sia a quanto ne è conseguito. E ciò fino appunto a riconoscergli, come sostenuto più sopra, anche il merito di aver creato alcune delle più importanti condizioni di sviluppo dei “Trent'anni Gloriosi” (1945-1975) del Welfare State. Un discorso, questo, che ritengo vada tanto più sostenuto, quanto più l'attuale ambiente culturale dominato com'è dall'anticomunismo neoliberale, tende a semplicemente a rimuovere o a svalutare come variante culturale di nicchia, da nostalgici inguaribili, quasi tutti i suoi argomenti più probanti.

Opporsi a questa rimozione a questa svalutazione non comporta per altro tacitare ogni riflessione critica sul come e il perché il comunismo del XX secolo sempre in questo stesso secolo (tra la seconda metà degli anni Settanta e la fine degli anni Ottanta) abbia finito per inabissarsi, per di più nel prevalente silenzio dei diretti interessati.

Anche a questo riguardo non posso non rinviare a L'enigma dell'ottobre1917, per una più estesa trattazione della questione, di cui qui avanzo comunque qualche ulteriore annotazione sempre a partire dal tema dell'organizzazione politica.

Da quanto già delineato più sopra, attorno all'ottobre 1917 il suo protagonista principale (i bolscevichi) passa dalla condizione di partito tra i partiti in seno ai soviet alla condizione, prima di milizia armata, poi Partito-Stato unico. Saltando al 1936 e alla Costituzione dell'Urss in Stato legale (successiva alle grandi purghe che interrompono definitivamente ogni retaggio bolscevico), si assiste ad un ulteriore passaggio organizzativo. Se nella configurazione che accoppiava Partito e Stato, quest'ultimo era più o meno contenuto nella dimensione del "mezzo Stato" (prescritta da Stato e Rivoluzione di Lenin), col 1936 esso si struttura invece in modo "organico", lasciando al partito una funzione più che altro ideologica e di propaganda. La strutturazione organica dello Stato implica allora un'esigenza di strutturazione economica della società sovietica. Un'esigenza che però viene vanificata dall'invasione nazista e dalla ben diversa esigenza, tutta politica, di organizzare quella che sarà la "Grande Guerra Patriottica".

Passando subito al dopoguerra, è molto significativo che uno degli ultimi testi di Stalin sia Problemi economici del socialismo (1952), titolo che la dice lunga su quanto le questioni politiche (di competenza del Partito) fossero oramai convertite in questioni economiche (di competenza della gigantesca, labirintica e crudele amministrazione statale sovietica). Ripensando al fatto che l'economicismo era la bestia nera del testo fondatore del bolscevismo (il Che fare? del 1902) si può immaginare che in mezzo secolo l'idea del comunismo sul finire dell'epoca di Stalin si stesse 
avviando alla conclusione di una sorta di ciclo evolutivo. Che il comunismo fosse una questione anzitutto economica è in effetti quanto si comincerà a sostenere dopo il XX congresso del Pcus nel 1956, quando oltre a denunciare il culto della personalità di Stalin si comincerà a parlare della concorrenza che il socialismo reale doveva ingaggiare col capitalismo per dimostrare quale sistema economico fosse più in grado di garantire il benessere dell'intera umanità. Quasi nessuno nei paesi capitalisti credette a questa visione ammansita e economicistica del comunismo, se è vero che la sua diffusione nel mondo di allora funzionò come deterrente nei confronti della congenita avidità del capitalismo. Anche tra i comunisti le perplessità non furono poche come si dimostrò nel corso del "lungo '68". Tuttavia il fatto che la patria della Rivoluzione Proletaria non desse più l'importanza dovuta alle passioni politiche necessarie per sfidare il capitalismo non poteva non minare alla radice la diffusione e l'incidenza mondiali di queste stesse passioni. E soprattutto non poteva non abbassare il livello intellettuale di tutte queste questioni.

Come ho sostenuto all'inizio di questo testo il guaio più grave della fine del comunismo non credo sia stata la fine in quanto tale, ma l'inadeguata elaborazione intellettuale del connesso lutto. A ciò sono convinto abbia contribuito proprio quell'economicismo tanto combattuto sia pur su piani ed in tempi diversi tanto da Lenin quanto da Althusser. 


\section{L'AUTORE}

Valerio ROMITELLI (1948) ha insegnato Metodologia delle Scienze Sociali e Storia dei Movimenti e dei Partiti Politici presso la Facoltà di Lettere e Filosofia dell'Università di Bologna. Tra i suoi libri: Gli dei che stavamo per essere (Bologna, Gedit, 2004); Etnografia del pensiero. Ipotesi e ricerche (Roma, Carocci, 2005); Fuori dalla società della conoscenza (Formigine, Infinito,2009); La felicità dei partigiani e la nostra. Organizzarsi per bande (Napoli, Cronopio, 2015).

URL: < http://www.studistorici.com/progett/autori/\#Romitelli > 